1. Coleman J, Brennan MF, Alektiar K, Russo P. Adult spermatic cord sarcomas: management and results. Ann Surg Oncol. 2003;10:669-75.

2. Peyrí E, Urban A, Martínez M, Sanmarti B. Liposarcoma dediferenciado del cordón espermático: degeneración de un lipoma previo. Actas Urol Esp. 2003;27:383-6.

3. Schwartz SL, Swierzewski III SJ, Sondak VK, Grossman HB. Liposarcoma of the spermatic cord: report of 6 cases and review of the literature. J Urol. 1995;153:154-7.

4. Soler J, Zuluaga A, Hidalgo M. Liposarcoma de cordón espermático: aportación de un nuevo caso y revisión de la literatura. Actas Urol Esp. 1999;23:447-54.

5. Henricks WH, Chu YC, Goldblum JR, Weiss SW. Dedifferentiated liposarcoma: a clinicopathological analysis of 155 cases with a proposal for an expanded definition of dedifferentiation. Am J Surg Pathol. 1997;21:271-81.
6. Calahorra F, Pérez C, Ramos A. Liposarcoma paratesticular del cordón espermático. Actas Urol Esp. 1990;3:202-4.

7. Garcia Morua A, Lozano Salinas JF, Valdes Sepulveda F. Liposarcoma of the espermatic cord: our experience and review of the literature. Actas Urol Esp. 2009;33:811-5.

8. Ballo MT, Zagars GK, Pisters PW. Spermatic cord sarcoma: outcome, patterns of failure and management. J Urol. 2001;166: 1306-10.

G. García-Fadrique*, E. Morán Pascual, G. Morales Solchaga, A. Soto, J.F. Morera y J.F. Jiménez-Cruz

Servicio de Urología, Hospital Universitario La Fe, Valencia, España

Autor para correspondencia.

Correo electrónico: gonzag1@hotmail.com

(G. García-Fadrique).

\title{
Anuria intermitente postrasplante renal secundaria a granuloma calcificado del neomeato
}

\section{Post-renal transplantation intermittent anuria secondary to calcified granuloma of the neomeatus}

\section{Sr. Director:}

Reportamos el caso de una paciente que presenta una obstrucción ureteral temprana secundaria a un granuloma calcificado de la unión ureterovesical después de un trasplante renal heterotópico derecho. No se ha descrito en la literatura esta variante de granuloma.

Se trata de una mujer de 34 años con insuficiencia renal crónica terminal (IRCT) secundaria a lupus eritematoso sistémico diagnosticado en 1990 que ingresa en el programa de hemodiálisis en el año 2000. Presenta antecedentes de hipersensibilización, hiperparatiroidismo secundario a IRC en tratamiento con Cinacalcet (Mimpara) y Calcitriol (Rocaltrol) e HTA en tratamiento con Atenolol, Doxazocina y Amlodipino.

Ingresó en marzo de 2008 para trasplante renal procedente de donante vivo relacionado (esposo). Se realiza, en el donante, nefrectomía izquierda por laparoscopia sin incidencias, obteniéndose un riñón de arteria y vena único y un uréter bien vascularizado y longitud correcta. Como parte del protocolo de desensibilización, la paciente recibió 2 dosis de Rituximab, 6 sesiones de inmunoabsorción pre trasplante así como 3 dosis de Gammaglobulina Monoclonal e inducción con Timoglobulina. Como tratamiento inmunosupresor posquirúrgico, Micofenolato Sódico $750 \mathrm{mg}$ v.o/12 h, Tacrolimus $6 \mathrm{mg}$ v.o/12 h, y Prednisona en esquema de disminución progresiva hasta $20 \mathrm{mg}$ v.o/d.

Se realizó el trasplante heterotópico en fosa renal derecha anastomosando la vena renal término lateral a la vena iliaca externa; la arteria renal a la arteria iliaca externa término lateral utilizando sutura continua de prolene 6 y 7/0 respectivamente con correcta perfusión tras desclampaje y sin complicaciones, realizando una implantación ureterovesical tipo Politano-Leabetter, anclando el extremo distal del uréter con 3 puntos sueltos de poliglecaprone (Monocril) 6/0, con diuresis inmediata. El tiempo de isquemia caliente fue de $2 \mathrm{~min}$ y de isquemia total fue de 50 min. Durante el postoperatorio, se realizó ecografía doppler renal que fue normal y un renograma isotópico con captación adecuada. La paciente presentó mejoría de la función renal con creatinina de $0,8 \mathrm{mg} / \mathrm{dl}$ al alta.

La paciente reingresó a los 14 días por dolor a nivel del injerto, anuria y deterioro de la función renal pasando la creatinina de 0,8 a 2,3 mg/dl. Se solicitó ecografía abdominal que informa de ectasia pieloureteral hasta la anastomosis ureterovesical donde se describe una imagen hiperecogénica compatible con litiasis. Reinicia diuresis de forma espontánea descendiendo los niveles de creatinina a $0,5 \mathrm{mg} / \mathrm{dl}$. Se complementa estudio de la lesión con TC abdominal que informa de ectasia pieloureteral del injerto renal y dilatación ureteral con la presencia de una masa calcificada intravesical en la zona de la anastomosis ureteral del injerto que podría corresponder a un hematoma o un granuloma calcificado (fig. 1). Se practicó citoscopia, evidenciando el neomeato calcificado.

Desde el punto de vista analítico, se objetivó hipercalcemia de 13,1 mg/dl (n: 8,4-10,2 mg/dl) en contexto de un hiperparatiroidismo secundario agravado por la suspensión semanas antes del Cinacalcet (Mimpara), el cual, tras medidas de 


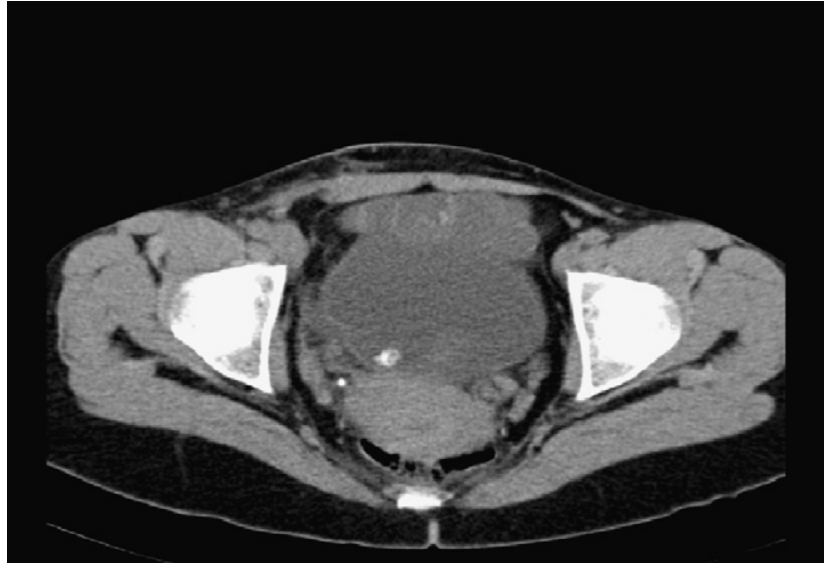

Figura 1 - TAC abdominal que informa de masa calcificada.

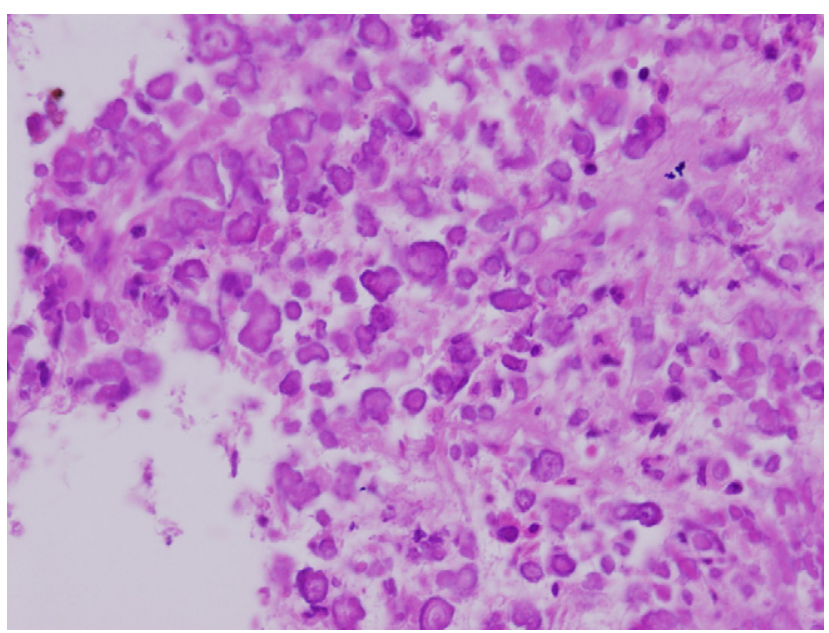

Figura 2 - Resultado de biopsia.

reposición hidroelectrolítica y manejo farmacológico, recuperó los niveles normales.

Dada la normalidad de las cifras de creatinina se decidió manejo expectante y control ambulatorio.

Ingresó nuevamente a los 3 días con deterioro de la función renal con creatinina de $2,2 \mathrm{mg} / \mathrm{dl}$ y anuria brusca de $10 \mathrm{~h}$. Se programó para nueva cistoscopia observándose neomeato calcificado y estenosado. Se llevó a cabo resección completa del granuloma calcificado y colocación de pigtail 4'8 Ch progresando $15 \mathrm{~cm}$. Se comprobó su correcta colocación radiológicamente y se dejó sonda Foley \#18, con orina clara al final de la intervención. Posteriormente, se normalizó la creatinina a niveles de $0,7 \mathrm{mg} / \mathrm{dl}$, dándose de alta en buenas condiciones y retirando el pigtail a los 30 días. La creatinina de control al mes fue $0,8 \mathrm{mg} / \mathrm{dl}$. El informe de biopsia reporta material fibrinoleucocitario y focos de necrosis y calcificación (fig. 2).

Las complicaciones de origen urológico en el trasplante renal representan la principal causa de morbilidad quirúrgica variando del 3 al $20 \%$ según las diferentes series ${ }^{1,2}$. Se han realizado diversas técnicas de reconstrucción del tracto urinario, siendo el tipo Politano-Leadbetter la que realizamos en nuestro centro desde que se inició el programa de trasplante renal en 1965, con muy buenos resultados y escasas complicaciones $^{3}$. La reconstrucción del neomeato se realiza en forma de «puño de camisa», evertiendo sus bordes y fijándolo con 3 puntos de sutura Monocril de 6/0.

La obstrucción de la vía y la fístula urinaria son las complicaciones urológicas más frecuentes. La técnica quirúrgica y la isquemia son los factores implicados en la presentación temprana, la isquemia y las infecciones en la fase tardía ${ }^{4}$. La obstrucción temprana ocurre en la primera semana, en el postoperatorio inmediato. Algunas de ellas son de extrema rareza, pero cuando se presentan, nos obligan a un tratamiento precoz. Las manifestaciones clínicas, así como la actitud terapéutica, dependerán del tipo de complicación. Las causas frecuentes son por hematoma, absceso, seroma, linfocele, edema, inflamación, coágulos y rotaciones del uréter o, como en este caso, granuloma calcificado del neomeato ${ }^{5}$. No hay publicaciones de granuloma calcificado a este nivel en la literatura. Los factores de riesgo implicados en este caso son la isquemia distal ureteral y el material de sutura de fijación (Monocril) que sirven de matriz para la cristalización y posterior crecimiento de la calcificación del granuloma potenciada por la hipercalciuria e hiperfosfaturia que están presentes en el hiperparatiroidismo secundario de la paciente, llamando la atención el proceso de formación tan rápido (26 días posquirúrgico).

El diagnóstico y tratamiento de esta complicación se realiza mediante cistoscopia intervencionista con buenos resultados y recuperación de la función renal.

B I B L I O G R A F Í A

1. Streeter EH, Little DM, Cranston DW, Morris PJ. The urological complications of renal transplantation: a series de 1535 patients. BJU Int. 2002;90:627-34.

2. Mundy AR, Podesta ML, Bewick M, Rudge CJ, Ellis FG. The urological complications of 1000 renal transplants. BJU. 1981; 53:397-402.

3. Ricart MJ. Obstrucción de la vía urinaria después del trasplante renal. Trasplante Renal. Barcelona: Ed. Torrag; 1983 206:

4. Faenza A, Nardo B, Catena F, Scolari MP, d'Arcangelo GL, Buscardi A, et al. Ureteral Stenosis after kidney transplantation. A study on 869 consecutive transplants. Transplant Int. 1999;12:334-40.

5. Gutiérrez del Pozo R, Mallafré Sala JM, Oliveira Alvés Jr J. Complicaciones de la cirugía antirreflujo. Estado Actual del tratamiento del reflujo vesico-ureteral. Asociación Española de urología. 1987.

G. Restrepo Madrid, R. Gutiérrez del Pozo, A. Trejo Rivas, L. Izquierdo y A. Alcaraz Asensio*

Servicio de Urología, Hospital Clinic de Barcelona, Barcelona, España

*Autor para correspondencia.

Correo electrónico: aalcaraz@clinic.ub.es (A. Alcaraz Asensio). 